\title{
Anomalous Relativistic Tunneling and Exotic Point Interactions
}

\author{
Pavel HejČík and Taksu Cheon \\ Laboratory of Physics, Kochi University of Technology, Tosa Yamada, Kochi 782-8502, Japan
}

\author{
PACS 03.65.Nk - Quantum scattering \\ PACS 03.65.Pm - Dirac equation \\ PACS 24.10.Jv - Nuclear relativistic dynamics
}

\begin{abstract}
We examine one-dimensional quantum scattering of a Dirac particle off relativistic potential barriers. With proper considerations of Dirac sea, existence of anomalous tunneling at zero incident-energy is revealed for a particular type of relativistic potential having same magnitudes and opposite signs for scalar and vector components. It is also shown that this leads to an exotic short range limit of the potentials.
\end{abstract}

Since its conception, Dirac equation has been plagued by difficulties related to the Dirac sea, and it is only after the proper taming of this monster of negative continuous spectra, that useful results are extracted from it. This fact is demonstrated again recently by two example, one on the resolution of Klein paradox [1,2], and the other on the resolution of Plesset's no-bound state problem [3-5]. In light of the resurgent interest in the Dirac equation in $>$ condensed matter physics [6,7], as well as of traditional interest in nuclear physics [8], we believe it is timely to report, in this Letter, a new result concerning a subtle effect of negative spectra on simple one dimensional po- tential scattering of Dirac particle off relativistic potential barriers.

Here, we show that proper consideration of Dirac sea has intriguing ramifications for low energy scattering matrices. - In particular, it is shown to cause anomalous tunneling at zero incident energy for " $S=-V$ potentials", a particular type of relativistic potential having the same magnitudes - and opposite signs for scalar and vector components of the potential.

We further show, by considering the short-range limit of this potential scattering, that it leads to exotic low-pass gaussian wave filter whose non-relativistic kinematics limit is "delta-prime" point interaction, that causes discontinuity in quantum wave function itself but not in its derivative [9], which is distinct from conventional delta-function point interaction.

We start by considering Dirac equation in one dimension that takes the following two-component form

$$
\left(\begin{array}{l}
\varphi^{\prime} \\
\chi^{\prime}
\end{array}\right)=\left(\begin{array}{cc}
0 & m+\varepsilon+S-V \\
m-\varepsilon+S+V & 0
\end{array}\right)\left(\begin{array}{l}
\varphi \\
\chi
\end{array}\right) .
$$
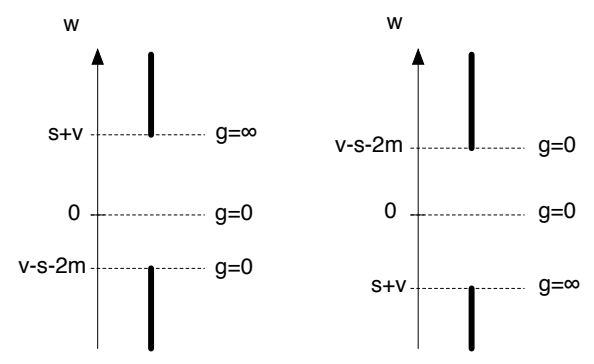

Fig. 1: Examples of Dirac spectra. The graph on the left depicts a case of $m>-s$, while the one on the right is of $m<-s$.

where $m, \varepsilon$ stand for the mass and relativistic energy of a Dirac particle, and $S$ and $V$ are scalar and (time component of) vector potentials. We only treat time-symmetric systems, so the spatial components of vector potential is absent. The prime signifies the spatial derivative $\frac{d}{d x}$.

We first consider one dimensional potential barrier of constant height located in positive $x$ region, formally given by

$$
V(x)=v \Theta(x), \quad S(x)=s \Theta(x),
$$

where $\Theta(x)$ is a Heaviside step function which is one for $x>0$ and zero for $x<0$. We define "mass excluded" energy $w$ by

$$
w=\varepsilon-m,
$$

which we assume to be positive. The spectra of a Dirac particle inside the potential barrier is composed of two disjoint continuous spectra separated by a gap. Some examples of the spectra are shown in FIG. 1. The scattering 
wave functions at $x<0$ and $x>0$ are given, respectively as

$$
\begin{aligned}
& \left(\begin{array}{l}
\varphi \\
\chi
\end{array}\right)=\left(\begin{array}{c}
1 \\
\frac{i k}{m+\varepsilon}
\end{array}\right) e^{i k x}-R\left(\begin{array}{c}
1 \\
\frac{-i k}{m+\varepsilon}
\end{array}\right) e^{-i k x}, \\
& \left(\begin{array}{l}
\varphi \\
\chi
\end{array}\right)=T\left(\begin{array}{c}
1 \\
\frac{i p}{m+\varepsilon+s-v}
\end{array}\right) e^{i p x},
\end{aligned}
$$

with the free momentum $k=\sqrt{\varepsilon^{2}-m^{2}}$ and the momentum $p=\sqrt{(\varepsilon-v)^{2}-(m+s)^{2}}$ inside the potential barrier. The expression (4) is valid for the case of continuous spectra for barrier region, $\varepsilon>|m+s|+v$. For the case of $|m+s|>\varepsilon>-|m+s|+v$, we need to make replacement, $p=i \kappa$ with $\kappa=\sqrt{-(\varepsilon-v)^{2}+(m+s)^{2}}$. The case of $\varepsilon<-|m+s|+v$ corresponds to a particle under the Dirac sea, for which a $p \rightarrow-p$ is needed in 4, but this case is soon to be sown irrelevant. Reflection and transmission rate is given by the squared absolute values of coefficients $R$ and $T$, respectively, when $p$ is real. When $\kappa$ is real, on the other hand, $T$ is the amplitude of wave function at classically forbidden region.

Expressing the momenta in terms of the energy $w$, we have

$$
\begin{aligned}
& k=\sqrt{w(w+2 m)}, \\
& p=\sqrt{(w-s-v)(w+2 m+s-v)} .
\end{aligned}
$$

The matching of wave functions (4) at $x=0$ gives

$$
1-R=T, \quad g(1+R)=T
$$

with

$$
g=\sqrt{\frac{w(w+2 m+s-v)}{(w+2 m)(w-s-v)}} Q=\frac{1}{i} \sqrt{\frac{w(w+2 m+s-v)}{(w+2 m)(s+v-w)}} Q .
$$

Here, $Q$ is the Giachetti-Sorace factor given by

$$
Q=1-\Theta(v-s-2 m-w) \Theta(s+v-w) .
$$

that represents the exclusion of wave function to the barrier region $x>0$ when the energy $w$ hits the negative energy spectra of Dirac equation with potentials $s$ and $v$. It is technically obtained from the proper connection condition $\varphi\left(0_{-}\right)=0, \chi\left(0_{-}\right)=$constant, which is obtainable as the $n \rightarrow \infty$ limit of $(x-1)^{n}$ potential, for which $\varphi\left(0_{-}\right)=0, \chi\left(0_{-}\right)=$constant is found to be the correct condition [5]. Fuller picture of this peculiar boundary condition may require the treatment of the problem with proper field theoretical setup as in [2], where the exclusion factor $Q$ could be understood as a result of many-body Pauli blocking.

The solution of the problem (6) is elementary, and we have

$$
T=\frac{2 g}{1+g}, \quad R=\frac{1-g}{1+g} .
$$

For large enough $w$, that satisfies the condition $w>$ $|m+s|-m+v$, the spectra inside the potential region
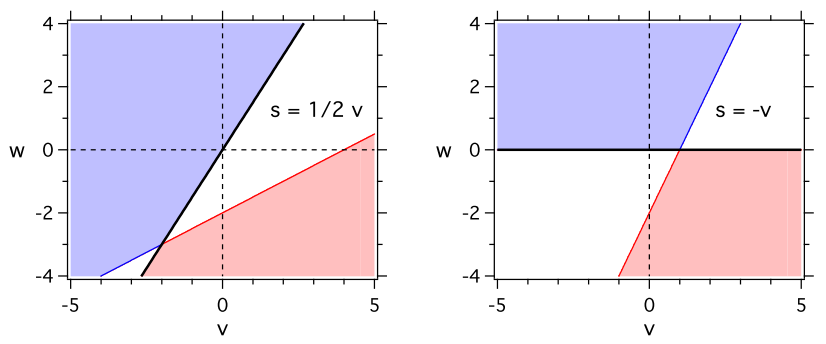

Fig. 2: Examples of Dirac spectra under scalar and vector potentials, $s$ and $v$. Here, $s$ is chosen to be proportional to $v$. The blue region represents the continuous particle spectra, while the red region is the "Dirac sea" spectra, which is occupied for the vacuum state. White region is the gap. Mass is set to be $m=1$. Among the lines separating spectra, solid line represents $w=v+s$, on which we have $g=\infty$ and the other line $w=v-s-2 m$ on which we have $g=0$. At the crossing point of these two lines, $g$ takes the value $g=\sqrt{\frac{v+s}{v-s}}$.

is continuous, and we have patial transmission and reflection specified by (9) with (7). We naturally have unitarity relation $|R|^{2}+|T|^{2}=1$. As we decrease $w$ down to the threshold energy $w=v-m+|m+s|, p$ approaches zero, and $g$ becomes either zero (if $s<-m$ and therefore $w=v-s-2 m$ ) or infinity (if $s>-m$ and therefore $w=v+s)$. They respectively correspond to perfect reflection $R=1$ with Dirichlet boundary $\varphi\left(0_{-}\right)=0$ or $R=-1$ with Neumann boundary $\chi\left(0_{-}\right)=0$.

Below this threshold, $|m+s|-m+v>w>-|m+s|-$ $m+v$ (or $|\varepsilon-v|<|m+s|$, if it occurs with positive $w$ ), we have exponential wave function with decay constant $\kappa$. The full reflection $|R|=1$ with quantum penetration $0<T<2$ to the classically forbiden area is observed. Note that there is no problem in having $|T|>1$ in this case, since the unitarity is guaranteed by decaying wave function $e^{-\kappa x}$. At the "Dirac sea" threshold, $w=v-m-$ $|m+s|, \kappa$ approaches zero, and $g$ becomes either infinity (if $s<-m$ and therefore $w=v+s$ ) or zero (if $s>-m$ and therefore $w=v-s-2 m$ ). They again respectively correspond to perfect reflection $R=-1$ with Neumann boundary $\chi\left(0_{-}\right)=0$ or $R=1$ with Dirichlet boundary $\varphi\left(0_{-}\right)=0$.

Below the Dirac sea threshold, $w<-|m+w|-m+v$ we have perfect reflection with Dirichlet condition $R=1$ as a result of Giachetti-Sorace factor.

When the energy $w$ approaches 0 , we have $\kappa \rightarrow 0$, that signifies the quantum penetration length to classically forbidden region $x>0$ becoming infinite. However, (7) tells us that we have $g=0$, and thus no penetration amplitude $T=0$ and, as a result, the perfect "classical" reflection $R=1$.

Above statements are true in generic case, depicted in the left hand graph in FIG. 2, for example, but there is an exception to the case when potentials $v$ and $s$ are related 


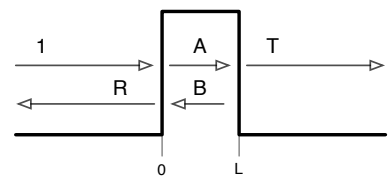

Fig. 3: Schematic representations of relativistic potential barrier scattering

by $s+v=0$, depicted in the right hand graph of FIG. 2 . In this case, there is a cancelation in the expression for $g$, (7) and we have

$$
g=\sqrt{\frac{w+2 m-2 v}{w+2 m}}=\frac{1}{i} \sqrt{\frac{2 v-w-2 m}{w+2 m}} .
$$

which is finite as a results of "merging" of $w=v+s$ line, on which $g=\infty$ holds, and $w=0$ line, on which $g=0$ holds.

This means that for this special case of opposite-sign but equal-magnitude scalar and vector potential, $s+v=0$, we have singular infinite-range penetration limit $\kappa \rightarrow 0$ with finite amplitude $0<|T|<2$ for zero energy barrier reflection $w \rightarrow 0$. We rush to note that this poses no paradox of any sort, since we still have full reflection $|R|=$ 1 albeit with some nontrivial phase for $R$. This is a subtle but an exotic exception, nonetheless, whose significance soon becomes obvious in the following.

We now consider one dimensional scattering by square well of constant height potential with spatial extension $L$ (FIG. 3), formally given by

$$
V(x)=v \Theta(x) \Theta(L-x), \quad S(x)=s \Theta(x) \Theta(L-x) .
$$

The scattering wave functions at $x<0,0<x<L$ and $L<x$ are given, respectively as

$$
\begin{aligned}
& \left(\begin{array}{l}
\varphi \\
\chi
\end{array}\right)=\left(\begin{array}{c}
1 \\
\frac{i k}{m+\varepsilon}
\end{array}\right) e^{i k x}-R\left(\begin{array}{c}
1 \\
\frac{-i k}{m+\varepsilon}
\end{array}\right) e^{-i k x}, \\
& \left(\begin{array}{l}
\varphi \\
\chi
\end{array}\right)=A\left(\begin{array}{c}
1 \\
\frac{i p}{m+\varepsilon+s-v}
\end{array}\right) e^{i p x}-B\left(\begin{array}{c}
1 \\
\frac{-i p}{m+\varepsilon+s-v}
\end{array}\right) e^{-i p x}, \\
& \left(\begin{array}{l}
\varphi \\
\chi
\end{array}\right)=T\left(\begin{array}{c}
1 \\
\frac{i k}{m+\varepsilon}
\end{array}\right) e^{i k x} .
\end{aligned}
$$

In a similar manner to the previous case, smooth connection conditions for both large and small components at $x=0$ and $x=L$ gives

$$
\begin{aligned}
& 1-R=A-B \\
& g(1+R)=A+B \\
& A e^{i p L}-B e^{-i p L}=T e^{i k L} \\
& A e^{i p L}+B e^{-i p L}=g T e^{i k L} .
\end{aligned}
$$

Elementary calculation yields the following expressions for transmission and reflection amplitudes;

$$
T=\frac{e^{-i k L}}{\cos p L-\frac{i}{2}(1 / g+g) \sin p L},
$$

$$
R=\frac{-\frac{i}{2}(1 / g-g) \sin p L}{\cos p L-\frac{i}{2}(1 / g+g) \sin p L} .
$$

This expression is literally valid for the energy $w>\mid m+$ $s \mid+v$. For the energy $|m+s|+v>w>-|m+s|+v$, we have to make replacement $p=i \kappa$ as before, which will result in the replacements $\cos p L \rightarrow \cosh \kappa L$ and $\sin p L \rightarrow$ $i \sinh \kappa L$ in (14). This expression is reduced to $T=0$, $R=1$ for the energy $w<-|m+s|+v$ with which we hit the Dirac sea spectra inside the potential barrier, where we have $Q=0$, thus $g=0$.

We look at the low energy limit of the scattering matrix $T$. Generically, for the case of $s \neq-v$, we have the quantity $g$ that approaches to zero as we take $w \rightarrow 0$ limit, causing the divergence of $1 / \mathrm{g}$, which guarantees the perfect reflection

$$
T \rightarrow 0, \quad R \rightarrow 1 \text { as } w \rightarrow 0 \quad(s+v \neq 0) .
$$

This simply is a exact expression of the intuitive statement that a generic obstacle works as a reflecting block for low energy projectile, or in other word, if we hit any barrier too slowly, we are bound to get reflected all the time.

However, for the special case of $s+v=0, g$ takes the form (10) after cancelation of $w$ in both denominator and enumerator of (7), and we have $g=$ finite and $\kappa \rightarrow 0$ (or $p \rightarrow 0$ ) as we take $w \rightarrow 0$ limit. We therefore obtain, from (14), a peculiar limit

$$
T \rightarrow 1, \quad R \rightarrow 0 \quad \text { as } w \rightarrow 0 \quad(s+v=0),
$$

which signifies an anomalous full transmission at zero energy. This is particularly intriguing for the case of decaying wave in the gap region, in which $\kappa$ is real, where decaying length $1 / \kappa$ becomes infinity at $w \rightarrow 0$ limit.

The situation is immediately understood by inspecting the illustrations in FIG. 4, Here, the graph in the left depicts a generic case that has normal perfect reflection at $w \rightarrow 0$ limit, while the graph in the right shows the anomalous zero energy transparency. The reason behind this transparency lies in the enhanced long range tunneling inside the barrier, which occurs because, at $w \rightarrow 0$, the energy approaches to the threshold of negative continuous spectra that exists right below $w=0$ for $s+v=0$ potentials. The presence of Dirac sea not only induces the perfect reflection for $w>0$ with $v>|s|$, for example, it also affects the decaying length and induces the anomalous tunneling, and transmission at $w \rightarrow 0$ limit for the case of $s+v=0$ potentials.

Let us now consider the $L \rightarrow 0$ limit of relativistic scattering. Straightforward limit will, of course, lead to disappearance of the barrier, $T \rightarrow 1$. Limit $L \rightarrow 0$ with constant volume integrals,

$$
v=\frac{\bar{v}}{L}, \quad s=\frac{\bar{s}}{L}, \quad(L \rightarrow 0),
$$

on the other hand, leads us to

$$
T=\frac{1}{\cos \beta+\frac{i}{2 \beta} \sin \beta[(\bar{v}+\bar{s}) / K+(\bar{v}-\bar{s}) K]} \Theta(\bar{v}+|\bar{s}|),
$$



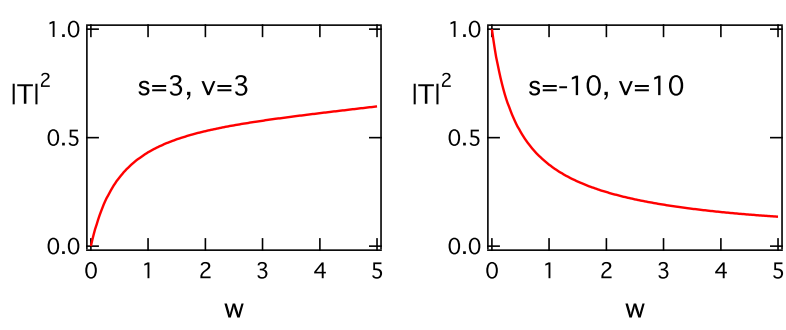

Fig. 4: Transmission rate as function of kinetic energy. The graph on the left is for normal case, and the one on the right is anomalous case, $s=-v$.

$$
\begin{gathered}
R=\frac{\frac{i}{2 \beta} \sin \beta[(\bar{v}+\bar{s}) / K-(\bar{v}-\bar{s}) K]}{\cos \beta+\frac{i}{2 \beta} \sin \beta[(\bar{v}+\bar{s}) / K+(\bar{v}-\bar{s}) K]} \Theta(-\bar{v}-|\bar{s}|) \\
+\Theta(\bar{v}+|\bar{s}|)
\end{gathered}
$$

with $\beta=\sqrt{\bar{v}^{2}-\bar{s}^{2}}=i \sqrt{\bar{s}^{2}-\bar{v}^{2}}$ and $K=\sqrt{\frac{w}{w+2 m}}$.

We can interpret this result in terms of relativistic point interactions specified by the boundary condition which is a most general time-reversal symmetric one [10]

$$
\left(\begin{array}{l}
\varphi\left(0_{+}\right) \\
\chi\left(0_{+}\right)
\end{array}\right)=\left(\begin{array}{cc}
\alpha & u_{-} \\
u_{+} & \alpha
\end{array}\right)\left(\begin{array}{l}
\varphi\left(0_{-}\right) \\
\chi\left(0_{-}\right)
\end{array}\right)
$$

with $\alpha^{2}-u_{+} u_{-}=1$. The scattering off the point interaction 190 is given

$$
T=\frac{1}{\alpha+\frac{i}{2}\left[u_{+} / K-u_{-} K\right]}, R=\frac{\frac{i}{2}\left[u_{+} / K+u_{-} K\right]}{\alpha+\frac{i}{2}\left[u_{+} / K-u_{-} K\right]},
$$

which allows the identifications

$$
u_{+}=(\bar{s}+\bar{v}) \frac{\sin \beta}{\beta}, \quad u_{-}=(\bar{s}-\bar{v}) \frac{\sin \beta}{\beta} .
$$

The special cases $\bar{s}=\bar{v}$ and $\bar{s}=-\bar{v}$ can be considered as the limiting cases of (18), and we have, for $\bar{s}=\bar{v}$

$$
T=\frac{1}{1+i \bar{v} / K}, \quad R=\frac{i \bar{v} / K}{1+i \bar{v} / K}
$$

while, for $\bar{s}=-\bar{v}$, we have

$$
T=\frac{1}{1+i \bar{v} K}, \quad R=\frac{-i \bar{v} K}{1+i \bar{v} K}
$$

If we take the non-relativistic limit in kinematics, $K \rightarrow$ $k /(2 m)$, these two cases are exactly identical to the scattering form delta and delta-prime point interactions [9], which represent high-pass and low-pass wave filters, respectively. Note that constructing non-standard point interactions, that results in (23), within non-relativistic framework involves highly singular procedures $[11,12]$.

Finally, we ask a question whether we can construct an analogue of the phenomena we have found in the framework of non-relativistic Schrödinger equation. We rewrite the Dirac equation (1) by eliminating small component $\chi$ in the form

$$
-\frac{d}{d x} \frac{1}{2 m^{*}} \frac{d}{d x} \varphi+U \varphi=w \varphi
$$

with effective mass $m^{*}$ and potential $U$ defined by

$$
m^{*}=m+\frac{w}{2}+\frac{S-V}{2}, \quad U=S+V .
$$

Assuming the conditions $w<<m$ and $|S-V|<<m$, we obtain Schrödinger equation with effective potential which is given by the sum of vector and scalar potentials. This is nothing but the true non-relativistic limit. However, we obtain non-standard low-energy limit by assuming the non-relativistic kinematics $w<<m$ in conjunction with strong relativistic potentials $|S-V| \sim m$. Specifically, we can reproduce anomalous transmission from Schrödinger equation (24) by setting $S=-V$ which results in $m^{*} \approx$ $m-V$ and $U=0$. This means that we can construct a purely non-relativistic model of anomalous scattering and delta-prime point interaction with just effective mass and no potential. Readers are warned, however, that this non-relativistic analogue scheme works only to an extent: When $S-V$ is negative in sign and so large, we obtain negative value for the effective mass $m^{*}$. For this bona fide relativistic dynamics, non-relativistic analogue (24) does not make sense, and therefore does not exist.

We have shown that a set of relativistic potentials having the property of same strength but opposite signs for scalar and vector components displays anomalous full tunneling and transparency at zero energy, while barrier starts functioning at higher energy. The short range limit of this phenomenon leads to a smooth relativistic realization of an exotic point interaction, delta-prime, that conventionally requires singular and esoteric constructions within nonrelativistic dynamics. It has been pointed out [13] that in three dimensions, the " $S=-V$ " relativistic potentials have an esoteric property called pseudospin symmetry [14] that has been found to play important role in the degeneracy structure of nuclear levels. Current work shows that there is yet another aspect to this pseudospin symmetric limit of relativistic potentials, which is revealed only in one dimensional systems.

We would like to thank Prof. Riccardo Giachetti for enlightening discussions. We also wish to thank Prof. Hiroshi Frusawa and Prof. Azhar Iqbal for useful comments. This work has been partially supported by the Grant-inAid for Scientific Research of Ministry of Education, Culture, Sports, Science and Technology, Japan under the Grant number 18540384. 


\section{REFERENCES}

[1] O. Klein, Die Reflexion von Elektronen an einem Potentialsprung nach der relativistischen Dynamik von Dirac, Z. Phys. 53 (1929) 157-165.

[2] P. Krekora, Q. Su and R. Grobe, Klein Paradox in Spatial and Temporal Resolution, Phys. Rev. Lett. 92 (2004) 040406.

[3] M.S. Plesset, The Dirac electron in simple fields, Phys. Rev. 41 (1932) 278-290.

[4] E.C. Titchmarsh, A problem in relativistic quantum mechanics, Proc. London Math. Soc. 11 169-192 (1961).

[5] R. Giachetti and E. Sorace, The discrete spectrum of the Dirac equation in confining potentials, arXiv: 0706.0127 (2007).

[6] A. Kholodenko and T. Vilgis, Elastic response of the Dirac chain, Phys. Rev. E50 (1994) 1257-1264.

[7] M.I. Katsnelson and K.S. Novoselov, Graphene: new bridge between condensed matter physics and quantum electrodynamics, Solid State Commun. 143 (2007) 3-13.

[8] B.D. Serot and J.D. Walecka, The Relativistic Nuclear Many-Body Problems, in Advances in Nuclear Physics Vol. 16, ed. by J.W. Negele and E. Vogt (Plenum, NY, 1986).

[9] P. Šeba, The generalized point interactionin one dimension, Czechoslovak J. Phys. 36 (1986) 667-673.

[10] S. Albeverio, F. Gesztesy, R. Høegh-Krohn and H. Holden, Solvable models in quantum mechanics: 2nd ed. with an appendix by P. Exner, (AMS Chelsea, 2005).

[11] J.E. Avron, P. Exner and Y. Last, Periodic Schrdinger operators with large gaps and Wannier-Stark ladders, Phys. Rev. Lett. 72, (1994) 896-899.

[12] T. Cheon and T. Shigehara, Realizing discontinuous wave functions with renormalized short-range interaction, Phys. Lett. A243 (1998) 111-116.

[13] J.N. Ginocchio, Pseudospin as a relativistic symmetry, Phys. Rev. Lett. 78 (1997) 436-439.

[14] A. Arima, M. Harvey and K. Shimizu, Pseudo LS coupling and pseudo SU3 coupling schemes, Phys. Lett. 30B (1969) 517-522. 